\title{
Chronic exposure of low dose salinomycin inhibits MSC migration capability in vitro
}

\author{
AGMAL SCHERZAD, STEPHAN HACKENBERG, KATRIN FROELICH, KRISTEN RAK, RUDOLF HAGEN, \\ JOHANNES TAEGER, MAXIMILLIAN BREGENZER and NORBERT KLEINSASSER
}

\author{
Department of Oto-Rhino-Laryngology, Plastic, Aesthetic and Reconstructive Head and Neck Surgery, \\ University of Wuerzburg, Wuerzburg, 97080 Bavaria, Germany
}

Received September 28, 2015; Accepted December 24, 2015

DOI: $10.3892 /$ br.2016.572

\begin{abstract}
Salinomycin is a polyether antiprotozoal antibiotic that is used as a food additive, particularly in poultry farming. By consuming animal products, there may be a chronic human exposure to salinomycin. Salinomycin inhibits the differentiation of preadipocytes into adipocytes. As human mesenchymal stem cells (MSC) may differentiate into different mesenchymal cells, it thus appeared worthwhile to investigate whether chronic salinomycin exposure impairs the functional properties of MSC and induces genotoxic effects. Bone marrow MSC were treated with low-dose salinomycin (100 nM) (MSC-Sal) for 4 weeks, while the medium containing salinomycin was changed every other day. Functional changes were evaluated and compared to MSC without salinomycin treatment (MSC-control). MSC-Sal and MSC-control were positive for cluster of differentiation 90 (CD90), CD73 and CD44, and negative for CD34. There were no differences observed in cell morphology or cytoskeletal structures following salinomycin exposure. The differentiation into adipocytes and osteocytes was not counteracted by salinomycin, and proliferation capability was not inhibited following salinomycin exposure. The migration of MSC-Sal was attenuated significantly as compared to the MSC-control. There were no genotoxic effects after 4 weeks of salinomycin exposure. The present study shows an altered migration capacity as a sign of functional impairment of MSC induced by chronic salinomycin exposure. Further in vitro toxicological investigations, particularly with primary human cells, are required to understand the impact of chronic salinomycin consumption on human cell systems.
\end{abstract}

Correspondence to: Dr Agmal Scherzad, Department of Oto-Rhino-Laryngology, Plastic, Aesthetic and Reconstructive Head and Neck Surgery, University of Wuerzburg, 11 Josef-Schneider-Strasse, Wuerzburg, 97080 Bavaria, Germany

E-mail: scherzad_a@ukw.de

Key words: human mesenchymal stem cells, salinomycin, migration, genotoxic effects, cell differentiation

\section{Introduction}

The ionophore salinomycin is a polyether antibiotic that is applied in veterinary medicine as an antiprotozoal agent (1). Specifically, the use of salinomycin in stockbreeding, particularly poultry, may have harmful side effects due to its severe toxicity. The data regarding human health hazards is poor; however, certain case reports show life-threatening side effects (2-4). By consuming animal products, there may be a chronic human exposure to salinomycin. Mortier et al (5) investigated egg samples from eight different countries in Europe for the presence of antibiotics and $20 \%$ of all samples were contaminated with salinomycin. Thus, the consequences on human health potentially caused by this chronic exposure to salinomycin appeared to warrant investigation. However, to the best of our knowledge, there are no studies on salinomycin intoxication following consumption of animal products. Szkudlarek-Mikh et al (6) reported that salinomycin inhibited the differentiation of preadipocytes into adipocytes, and that salinomycin was the most potent inhibitor. This was not due to apoptosis or inhibition of cell proliferation, but was closely associated with suppression of the transcriptional activity of adipogenesis. The latter was explained by the suppression of the CCAAT/enhancer-binding proteins and the peroxisome proliferator-activated receptor (6). This result raises the question of whether chronic salinomycin exposure interferes with the differentiation capability of human cells, such as mesenchymal stem cells (MSC) as well. MSC are undifferentiated cells and there are no specific markers for their identification. According to the International Society of Cellular Therapy, MSC have to meet the following criteria: Adherent growth behavior, be positive for cluster of differentiation (CD) 105, CD90 and CD73 and negative for CD14, CD11b and HLAII cell surface markers. Furthermore, MSC must have the ability to differentiate into bone, cartilage or fat $(7,8)$.

In our previous study, MSC were exposed to salinomycin for $24 \mathrm{~h}$. Salinomycin did not affect any essential properties of MSC. However, dose-dependent cytotoxic effects of salinomycin on MSC were observed. In the present study, the following functional impairments of MSC following chronic salinomycin exposure were examined: Alteration of cell surface markers and differentiation capability, genotoxicity, migration, proliferation capacity and cytoskeletal architecture. 


\section{Materials and methods}

MSC isolation and salinomycin treatment. MSC were isolated from the human bone marrow of 5 voluntary patients undergoing surgery in the Department of Orthopedic Surgery, University of Wuerzburg. The study was approved by the Ethics Committee of the Medical Faculty, University of Wuerzburg (12/06; Bavaria, Germany), and informed consent was obtained from all the individuals included. The isolation of MSC was performed according to previous studies using Ficoll density gradient centrifugation ( $30 \mathrm{~min}, 227 \mathrm{x}$ g, density $=1,077 \mathrm{~g} / \mathrm{ml}$; Biochrom AG, Berlin, Germany) $(9,10)$. Subsequent to the collection of the cells from the interphase, several washing steps with phosphate-buffered saline (PBS) (Roche Diagnostics GmbH, Mannheim, Germany) containing 2\% fetal calf serum (FCS) (Linaris, Wertheim-Bettingen, Germany) were performed. The isolated cells were resuspended in Dulbecco's modified Eagle's medium-expansion medium (DMEM-EM) (Gibco Invitrogen, Thermo Fisher Scientific, Karlsruhe, Germany) with $10 \%$ FCS, $1 \%$ penicillin/streptomycin (Sigma-Aldrich, Schnelldorf, Germany). After incubation at $37^{\circ} \mathrm{C}$ and $5 \% \mathrm{CO}_{2}$ in DMEM-EM overnight, the tissue culture plates were washed to remove residual non-adherent cells. Every other day the medium was changed. Cell morphology was investigated by inverted microscopy (Leica DMI 4000B Inverted Microscope; Leica Microsystems, Wetzlar, Germany). MSC were treated with $100 \mathrm{nM}$ of salinomycin for 4 weeks (MSC-Sal). The medium containing $100 \mathrm{nM}$ salinomycin was changed every other day. MSC cultivated in DMEM-EM served as the control (MSC-control).

Expression of the cell surface marker. The cell surface profile of MSC-Sal was investigated by flow cytometry (BD FACSCanto $^{\mathrm{TM}}$; BD Biosciences, Heidelberg, Germany) as follows: Before cells were incubated on ice with $5 \%$ FCS for $1 \mathrm{~h}$, they were first trypsinized and washed with PBS. Subsequently, the cells were washed with PBS and incubated with anti-CD90 (cat no. 559869), anti-CD73 (cat no. 550257), anti-CD44 (cat no. 555478), and anti-CD34 (cat no. 555820; all from BD Biosciences). The results were compared to MSC-control.

Multi-differentiation capacity. MSC-Sal were incubated with adipogenic medium, which consisted of DMEM-EM containing $10 \mu \mathrm{M}$ dexamethasone (Sigma-Aldrich), and $10 \mathrm{ng} / \mathrm{ml}$ recombinant human insulin. The osteogenic medium contained DMEM-EM, $10^{-7} \mathrm{M}$ dexamethasone, $10 \mathrm{mM}$ $\beta$-glycerophosophate, $50 \mu \mathrm{g} / \mathrm{ml}$ ascorbic acid-2-phosphate (all from Sigma-Aldrich). Cells were incubated for 3 weeks. The medium was changed every third day. The differentiation potential was examined by histological staining. Oil Red O was conducted to demonstrate the adipogenic differentiation. The von Kossa method was used to confirm osteogenic differentiation. For gene expression analysis, reverse transcription-quantitative polymerase chain reaction (RT-qPCR) was conducted. In this technique, the expression of specific marker genes for osteogenic and adipogenic differentiation was evaluated. All the samples were analyzed in triplicate for each patient. Total RNA was extracted using the RNAeasy Mini kit (Qiagen, Hilden, Germany), following the manufacturer's instructions. Subsequently, total RNA was reverse transcribed to cDNA using the high-capacity RNA-to-cDNA Master Mix (Applied Biosystems, Darmstadt, Germany). The RT-qPCR device (Applied Biosystems) was used. The PCR primers were purchased from Life Technologies (Darmstadt, Germany). For adipogenic differentiation gene expression of lipoprotein lipase [LPL; accession number (AN): NM_000237.2] and leptin (LEP; AN: NM_002303.5) were measured. The osteogenic differentiation was confirmed by the analysis of alkaline phosphatase (ALP; AN: NM_000478.4), bone $\gamma$-carboxyglutamate protein (BGLAP; AN: NM_199173.4) and runt-related transcription factor 2 (RUNX-2/cbfa-1; NM_004348.3). The amplifications for gene quantification were: $50^{\circ} \mathrm{C}$ for $2 \mathrm{~min}$; $95^{\circ} \mathrm{C}$ for $10 \mathrm{~min}$, and 40 cycles at $95^{\circ} \mathrm{C}$ for $15 \mathrm{sec}$ and $60^{\circ} \mathrm{C}$ for $1 \mathrm{~min} . \Delta \mathrm{CT}$ values are presented as relative quantification, which were normalized to the gene expression of the reference gene GAPDH (AN: NM_002046.3).

Cell migration. The scratch assay was used to clarify a possible alteration in MSC migration induced by chronic exposure to $100 \mathrm{nM}$ salinomycin. MSC cells $\left(1 \times 10^{5}\right.$ cells $\left./ \mathrm{ml}\right)$ were cultivated in a 12 -well round bottom plate at $37^{\circ} \mathrm{C}$. After $24 \mathrm{~h}$, a straight-line wound was induced with a sterile 1-ml pipette tip. Following this, culture plates were washed with PBS, images were captured $(t=0 \mathrm{~h})$ and incubated for another $24 \mathrm{~h}$ at $37^{\circ} \mathrm{C}$ with $5 \% \mathrm{CO}_{2}$. To analyze the relative migration, images of the plates were captured $(\mathrm{t}=24 \mathrm{~h}$ ) (Leica DMI 4000B Inverted Microscope) and the percentage of the wound closure was evaluated. The area between the wound borders at time $\mathrm{t}=24 \mathrm{~h}$ was defined as the percentage of wound closure compared to the wound borders at time $\mathrm{t}=0 \mathrm{~h}$. The calculation was investigated using ImageJ (version 1.43u). MSC cultivated with DMEM-EM served as the control. The experiment was performed in triplicate using MSC of all patients $(\mathrm{n}=5)$.

Immunocytochemical analysis of $\alpha$-tubulin. MSC-Sal were washed several times with PBS and fixed with 4\% paraformaldehyde for $30 \mathrm{~min}$ at room temperature (RT). Another 5 min of fixation with acetone at RT was performed. Subsequently, cells were incubated with $10 \%$ bovine serum albumin (BSA) (Carl Roth $\mathrm{GmbH}$, Karlsruhe, Germany) in Tris-buffered saline $200 \mathrm{mM}$ Tris-base (pH 8), 8\% NaCl and $1 \%$ Tween-20 (TBS-T) (all from Sigma-Aldrich). Incubation of MSC in TBS-T containing 1\% BSA plus a mouse monoclonal antibody against $\alpha$-tubulin (1:500; Sigma-Aldrich) was followed for $10 \mathrm{~h}$. After three washing steps with TBS-T (each step $5 \mathrm{~min}$ ), MSC were incubated for $1 \mathrm{~h}$ in $1 \% \mathrm{BSA}$ at RT with Alexa 488 goat anti-mouse secondary antibody 1:500 (Gibco Invitrogen) and $5 \mathrm{mg} / \mathrm{ml}$ DAPI (Sigma-Aldrich). A fluorescence microscope (Leica DMI 4000B Inverted Microscope) was used for cell examination.

Proliferation analysis. The expression of Ki67 was investigated via immunocytochemical analysis and RT-qPCR. A mouse monoclonal antibody against Ki67 (1:500; Sigma-Aldrich) was used. The gene expression of Ki67 (AN: NM_002417.4) (Applied Biosystems) was measured by RT-qPCR.

Genotoxicity. The alkaline single-cell microgel electrophoresis (comet) assay was performed for verification, for 

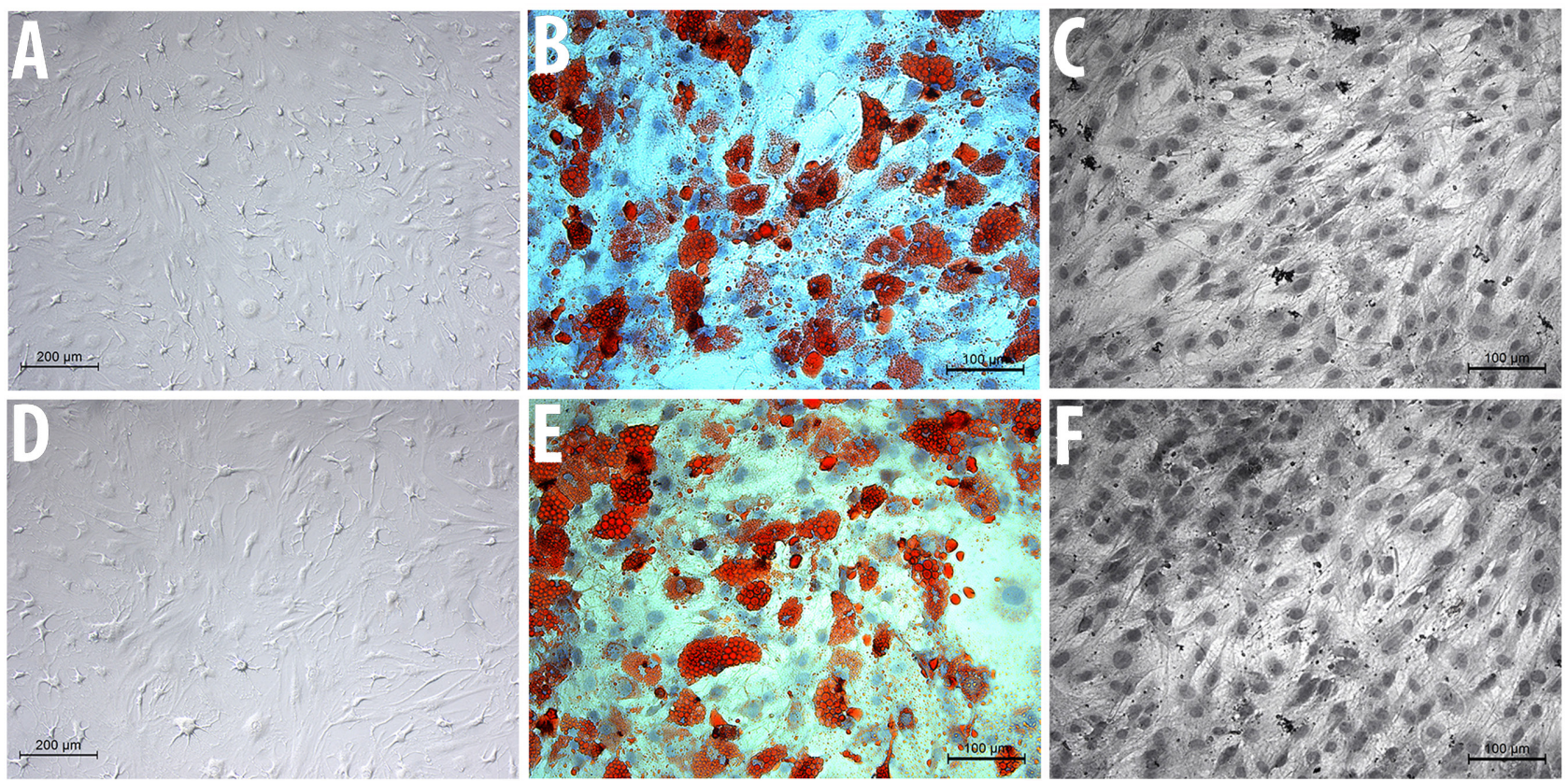

Figure 1. Microscopic analysis of (A) MSC-control and (D) MSC-Sal. The differentiation of (B) MSC-control and (E) MSCSal into adipocytes is illustrated with Oil Red O staining, and shows the intracellular lipid droplets. The differentiation of (C) MSC-control and (F) MSC-Sal into osteocytes was measured by van Kossa staining and characterizes mineralization by marking the mineral components. MSC, mesenchymal stem cells.

example, of possible DNA strand breaks. MSC were treated with $100 \mathrm{nM}$ of salinomycin for 4 weeks. The slides were prepared as previously described by Buehrlen et al (11). The slides were observed by fluorescence microscopy (magnification, $x 400$; DMLB Leica Microsystems) with the following filters: A green excitation filter $(515-560 \mathrm{~nm}$ band pass), a dichromatic beam splitter (580 $\mathrm{nm}$ long pass), and an emission filter (590 nm long pass). In every sample, 100 cells were counted. For the DNA fragmentation analysis, the KOMET 5.5 image system (Kinetic Imaging, Liverpool, UK) was used. Tail DNA (TD), tail length (TL) and Olive tail moment (OTM) as a product of the median migration distance and the percentage of DNA in the tail were measured, as previously described by Olive et al (12). The comet assay was performed for all MSC-Sal $(n=5)$ and MSC-control $(n=5)$.

Statistical analysis. All the data were transferred to standard spreadsheets and analyzed by statistical analysis (GraphPad Prism 5.0 software; GraphPad, Inc., La Jolla, CA, USA). The Kruskal-Wallis test was carried out to evaluate statistical significance. $\mathrm{P}<0.05$ was considered to indicate a statistically significant difference and are shown by an asterisk.

\section{Results}

Cell morphology and multidifferentiation capacity. The two cell groups, MSC-Sal and MSC-control, maintained their typical spindle-shaped fibroblast-like morphology. Salinomycin did not induce any signs of apoptosis or senescence. The cell surface analysis revealed that MSC-Sal and MSC-control were positive for CD90, CD73 and CD44, and negative for CD34 (Fig. 1).

MSC-Sal and MSC-control were cultivated in osteogenic and adipogenic medium. The multidifferentiation capacity was

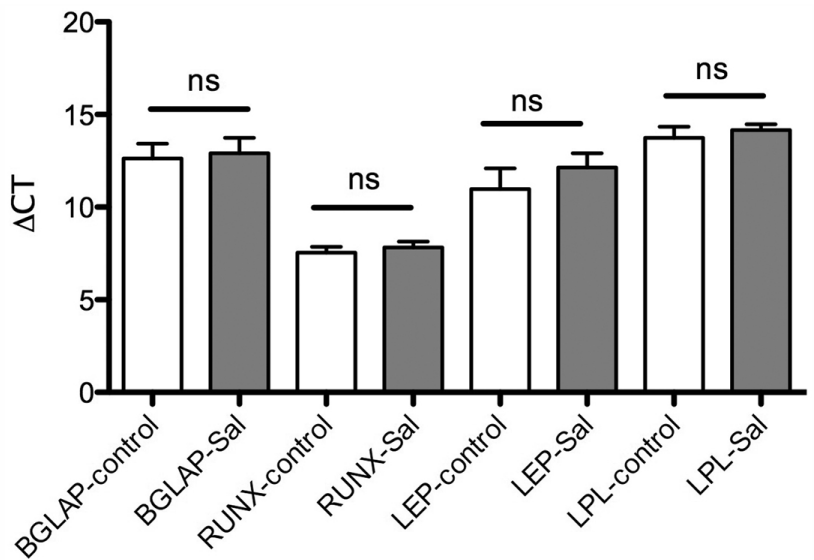

Figure 2. Analysis of gene expression with RT-qPCR. MSC-Sal and MSC-control were differentiated into adipocytes and osteocytes. The differentiations were confirmed with RT-qPCR. Salinomycin did not alter the gene expression for adipogenic and osteogenic differention as compared to the control. RT-qPCR, reverse transcription-quantitative polymerase chain reaction; MSC, mesenchymal stem cells; BGLAP, bone $\gamma$-carboxyglutamate protein; RUNX-2, runt-related transcription factor 2; LEP, leptin; ALP, alkaline phosphatase; LPL, lipoproteinlipase; ns, not significant.

examined via staining methods, as well as with RT-qPCR. The Oil Red O and the von Kossa staining showed that MSC-Sal and MSC-control were able to differentiate into adipocytes and osteocytes. These results were confirmed by RT-qPCR. The gene expression for osteogenic and adipogenic differentiation was detectable in MSC-Sal and MSC-control following cultivation in adipogenic and osteogenic medium. The $\Delta \mathrm{CT}$ values showed no significant differences (Fig. 2).

Migration capability. The scratch assay was used to determine a possible influence of chronic salinomycin exposure on MSC migration. After a repair period of $24 \mathrm{~h}$ at $37^{\circ} \mathrm{C}$, 

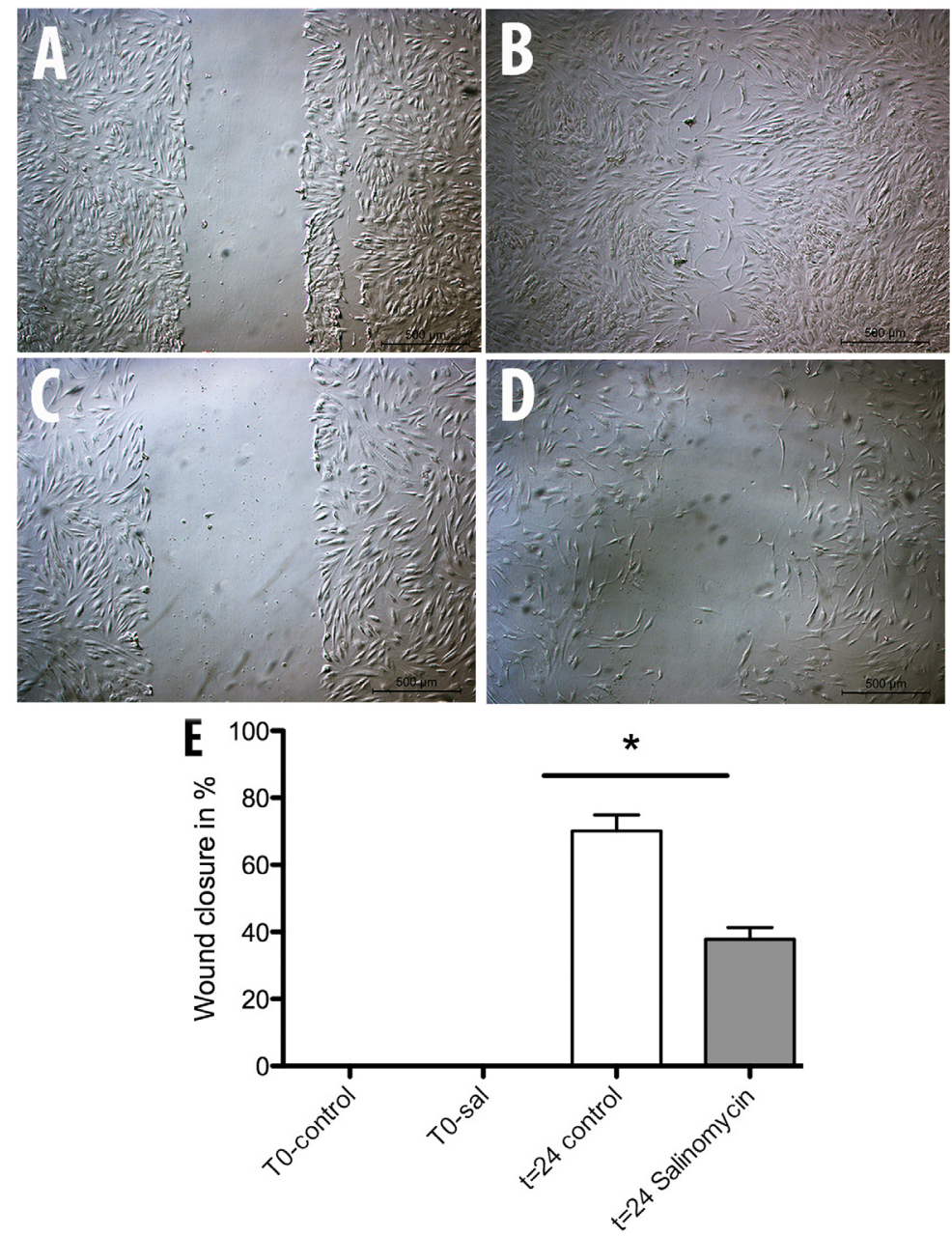

Figure 3. Analysis of (A and B) MSC-control and (C and D) MSC-Sal migration capability. A wound was generated at time (A and C) $t=0 \mathrm{~h}$. After $24 \mathrm{~h}$ the areas of wound closure were measured with the program ImageJ. The statistical evaluaton revealed a significant attenuation of migration capability in (E) MSC-Sal. MSC, mesenchymal stem cells. " $\mathrm{P}<0.05$.
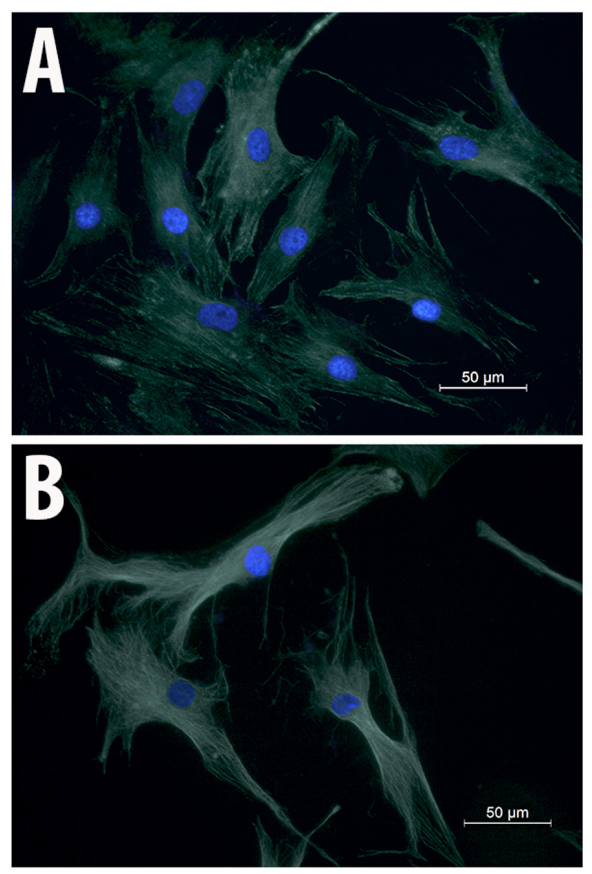

Figure 4. Immunohistochemical analysis of (A) MSC-control and (B) MSC-Sal using $\alpha$-tubulin antibodies. The cytoskeletal structure of MSC-Sal was not altered by salinomycin. MSC, mesenchymal stem cells. the wound area was controlled and the level of wound closure measured. The assay showed a significant inhibition of MSC-Sal ability in closing the wound area compared to the MSC-control (Fig. 3). MSC-control induced 70\% wound closure while MSC-Sal was only able to show a wound closure of $40 \%$.

Immunocytochemical analysis of $\alpha$-tubulin. To examine whether the migration impairment induced by salinomycin is associated with changes in MSC cytoskeletal structure, immunocytochemical analysis of $\alpha$-tubulin was performed. MSC-Sal and MSC-control showed no effect on their cell cytoskeletal structures. There were no signs for disruption of the tubulin fibers or structural instability in MSC-Sal and MSC-control. The shapes of the two cell types (MSC-Sal and MSC-control) did not show any differences (Fig. 4).

Proliferation analysis. The expression of Ki67 was investigated to evaluate possible changes in the activity of cell proliferation. The immunohistochemical analysis of Ki67 revealed no differences between MSC-Sal and MSC-control. The results were confirmed by RT-qPCR. $\Delta$ CT values in Fig. 5 show no statistical significant difference between MSC-Sal and MSC-control (Fig. 6). 


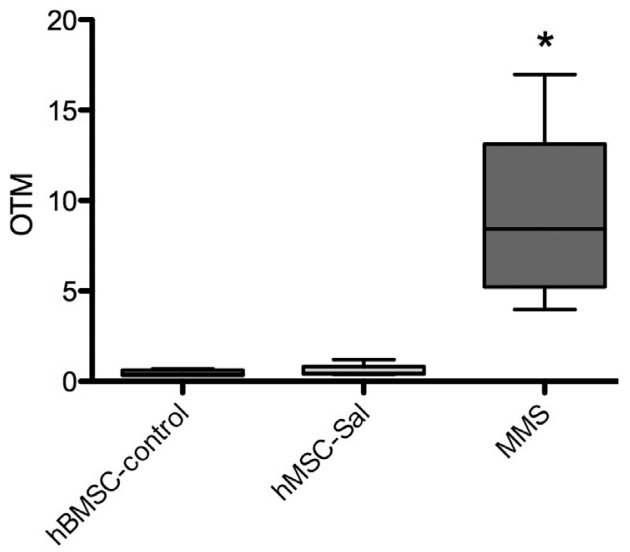

Figure 5. Comet assay was performed in MSC-control and MSC-Sal to search for genotoxic effects. In all samples, no significant increase in the Olive tail moment (OTM) was observed. MMS served as a positive control. MSC, mesenchymal stem cells; MMS, methyl methane sulfonate. ${ }^{*} \mathrm{P}<0.05$.
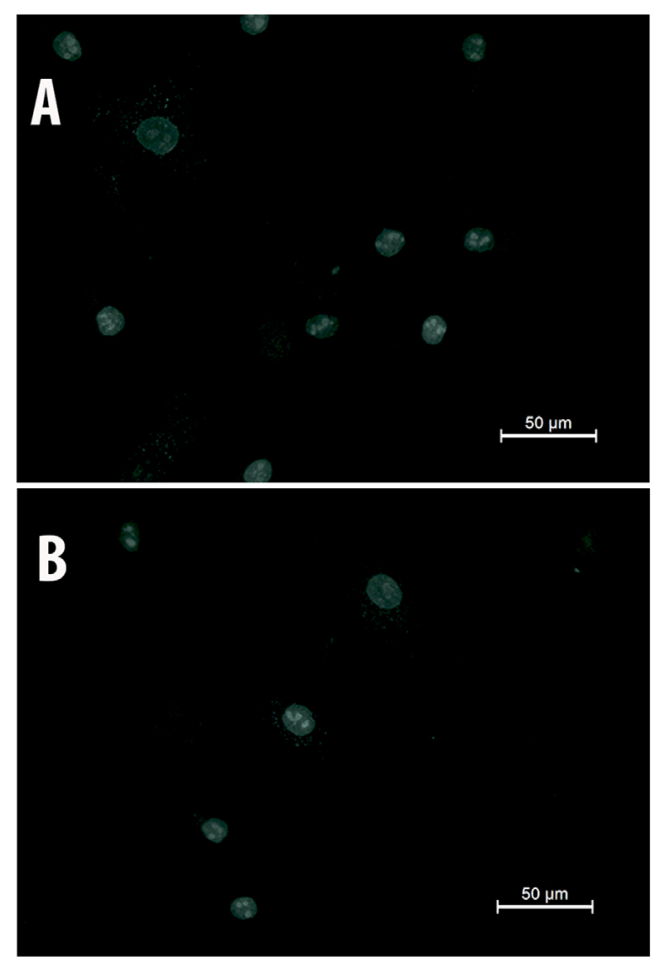

C

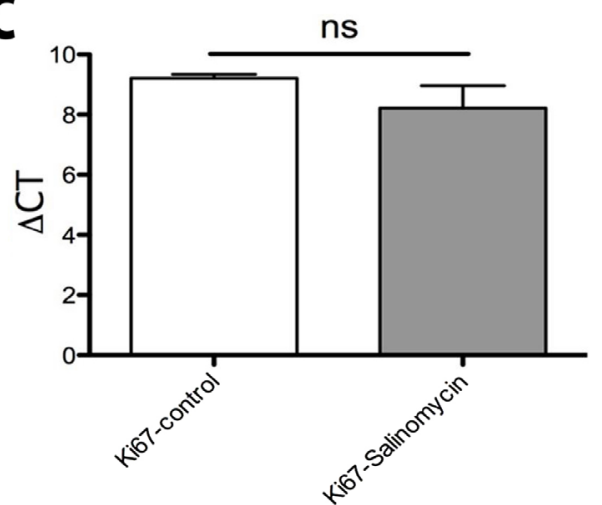

Figure 6. Immunhistochemistry staining of cell nuclei. (A) MSC-control and (B) MSC-Sal nuclei were positive for Ki67. (C) Reverse transcription-quantitative polymerase chain reaction showed no significant differences in Ki67 gene expression between MSC-control and MSC-Sal. MSC, mesenchymal stem cells.
Genotoxicity. The comet assay was performed in MSC-Sal and MSC-control. In all samples, no significant increase in the OTM, as an indicator for genotoxic effects, was observed. MMS as a positive control induced a significant increase in DNA breakage. Data are presented in Fig. 5.

\section{Discussion}

Ionophores, such as salinomycin, induce toxicity by increasing the intracellular sodium concentration, which consecutively increases the intracellular $\mathrm{Ca}^{2+}$ concentration due to the $\mathrm{Na}^{+} / \mathrm{Ca}^{2+}$ exchange in the plasma membrane and mitochondria (13). This proton exchange leads to an alteration in $\mathrm{pH}$, and thereby increases osmotic pressure inside the cell, which may lead to apoptosis (14). Ionophores show a broad spectrum of biological activity, such as antibacterial activity, particularly for Gram-positive bacteria and also including antibiotic-resistant $S$. aureus, as well as antiviral and antiparasitic activity (15). In target animal species, such as poultry, salinomycin did not exhibit any toxic side effects. The toxicity of ionophores, such as monensin, has also been investigated. Todd et al (16) could identify acute toxic symptoms, such as anorexia, hypoactivity, skeletal muscle weakness, ataxia, diarrhea, decreased weight gain and delayed fatalities induced by monensin. Chronic exposure also resulted in anorexia, weakness, ataxia and increased serum muscle enzyme combined with severe skeletal muscle degeneration and necrosis (16). The data concerning salinomycin toxicity in humans is extremely poor and divergent. In a clinical pilot study of a patient with metastatic invasive ductal breast cancer, systemic salinomycin application of $200 \mu \mathrm{g} / \mathrm{kg}$ every other day was tolerated extremely well (17), and resulted in a significant reduction in the cancer. The same effects were observed in patients suffering from head and neck cancer as well as from ovarian cancer. The side effects of intravenous salinomycin following administration were tachycardia and mild tremor (17). By contrast, there are certain case reports postulating harmful side effects induced by salinomycin, such as rhabdomyolysis and renal failure $(2,4)$. These divergences between the results of case reports and clinical pilot studies may be due to differences in the dosage of salinomycin. In our recent study, 10-20 $\mu \mathrm{M}$ salinomycin induced cytotoxic effects in human peripheral lymphocytes and nasal mucosa cells in vitro (18). However, no genotoxic effects were detectable at subcytotoxic levels. In the clinical studies, the dosage of salinomycin ranged from $200-250 \mu \mathrm{g} / \mathrm{kg}$. All the patients experienced a reduction in the cancer, but complete remission was not achieved. Hypothetically, this may be due to the dosages of salinomycin applied. Higher amounts of salinomycin could have a more potent effect on the cancer. Story and Doube (2) described an accidental ingestion of salinomycin by a farmer. The dosage was not determined, however, it was estimated that $1 \mathrm{mg} / \mathrm{kg}$ of the body weight was swallowed. This resulted in life-threatening neuropathia, rhabdomyolysis and a 6-week hospitalization (2). Therefore, the biological hazards in humans induced by salinomycin have to be explored in greater detail as well.

In our previous study, acute exposure of salinomycin to MSC revealed a dose-dependent attenuation of cell viability without affecting the cell migration capability (19). The ability of MSC to differentiate into osteocytes and adipocytes 
remained unchanged after treatment with salinomycin for $24 \mathrm{~h}$. As chronic exposure affects the differentiation of adipocytes, it was worthwhile to investigate whether chronic exposure of salinomycin inhibits the multipotency of MSC as well. In the present study, the staining methods and gene expression analysis revealed no alteration in MSC multipotency following chronic salinomycin exposure as compared to the control group. The cell cytoskeletal structure was also not affected. Notably, however, the migration capability of MSC attenuated significantly following chronic exposure to salinomycin. One possible explanation of this phenomenon may be the regulation of the C-X-C chemokine receptor type 4 (CXCR4) by salinomycin. CXCR4 is a ligand of the chemokine stromal-derived factor-1, and both have an important role in the mediation of MSC migration (20). Liu et al (21) demonstrated an enhancement of MSC migration following gene modification of MSC with CXCR4. Larzaba et al (22) investigated the effects of salinomycin on lung primary tumors and metastasis, and in this study, salinomycin reduced the expression of CXCR4 significantly. CXCR4 is highly expressed by MSC within the bone marrow and is markedly reduced during in vitro expansion (23). The chronic exposure of salinomycin may have induced a reduction in CXCR4. The evaluation of CXCR4 and possible shortcomings of the present study will be addressed in future studies: First, an exposure of 4 weeks with salinomycin may be too short to detect side effects. Second, MSC have a considerable ability to restore their biological behavior. In the present study MSC were treated for 4 weeks with salinomycin. Following this period they were cultured in expansion medium with supplements either for adipogenic or osteogenic differentiation. During the differentiation process, MSC were not exposed to salinomycin. This may have had an impact on the regeneration of the differentiation capability.

In conclusion, salinomycin induced a significant attenuation of MSC migration, while differentiation, as well as cell morphology and proliferation, were unchanged. Further investigations, for example with different primary human cells, are required in order to evaluate the potential cytotoxic effects of acute and chronic salinomycin exposure.

\section{References}

1. Boehmerle W and Endres M: Salinomycin induces calpain and cytochrome c-mediated neuronal cell death. Cell Death Dis 2: e168, 2011

2. Story $P$ and Doube A: A case of human poisoning by salinomycin, an agricultural antibiotic. N Z Med J 117: U799, 2004.

3. Caldeira C, Neves WS, Cury PM, Serrano P, Baptista MA and Burdmann EA: Rhabdomyolysis, acute renal failure and death after monensin ingestion. Am J Kidney Dis 38: 1108-1112, 2001.

4. Kouyoumdjian JA, Morita MP, Sato AK and Pissolatti AF: Fatal rhabdomyolysis after acute sodium monensin (Rumensin) toxicity: Case report. Arq Neuropsiquiatr 59: 596-598, 2001.

5. Mortier L, Huet AC, Charlier C, Daeseleire E, Delahaut P and Van Peteghem C: Incidence of residues of nine anticoccidials in eggs. Food Addit Contam 22: 1120-1125, 2005.

6. Szkudlarek-Mikho M, Saunders RA, Yap SF, Ngeow YF and Chin KV: Salinomycin, a polyether ionophoric antibiotic, inhibits adipogenesis. Biochem Biophys Res Commun 428: 487-493, 2012.
7. Dominici M, Le Blanc K, Mueller I, Slaper-Cortenbach I, Marini F, Krause D, Deans R, Keating A, Prockop Dj and Horwitz E: Minimal criteria for defining multipotent mesenchymal stromal cells. The international society for cellular therapy position statement. Cytotherapy 8: 315-317, 2006.

8. Horwitz EM, Le Blanc K, Dominici M, Mueller I, Slaper-Cortenbach I, Marini FC, Deans RJ, Krause DS and Keating A; International Society for Cellular Therapy: Clarification of the nomenclature for MSC: The international society for cellular therapy position statement. Cytotherapy 7 : 393-395, 2005.

9. Lee RH, Kim B, Choi I, Kim H, Choi HS, Suh K, Bae YC and Jung JS: Characterization and expression analysis of mesenchymal stem cells from human bone marrow and adipose tissue. Cell Physiol Biochem 14: 311-324, 2004.

10. Scherzed A, Hackenberg S, Froelich K, Kessler M, Koehler C, Hagen R, Radeloff A, Friehs G and Kleinsasser N: BMSC enhance the survival of paclitaxel treated squamous cell carcinoma cells in vitro. Cancer Biol Ther 11: 349-357, 2011.

11. Buehrlen M, Harreus UA, Gamarra F, Hagen R and Kleinsasser NH: Cumulative genotoxic and apoptotic effects of xenobiotics in a mini organ culture model of human nasal mucosa as detected by the alkaline single cell microgel electrophoresis assay and the annexin $\mathrm{V}$-affinity assay. Toxicol Lett 169: 152-161, 2007.

12. Olive PL, Durand RE, Le Riche J, Olivotto IA and Jackson SM: Gel electrophoresis of individual cells to quantify hypoxic fraction in human breast cancer. Cancer Res 53: 733-736, 1993.

13. Dorne JL, Fernández-Cruz ML, Bertelsen U, Renshaw DW, Peltonen K, Anadon A, Feil A, Sanders P, Wester P and Fink-Gremmels J: Risk assessment of coccidostatics during feed cross-contamination: Animal and human health aspects. Toxicol Appl Pharmacol 270: 196-208, 2013.

14. Antoszczak M, Popiel K, Stefańska J, Wietrzyk J, Maj E, Janczak J, Michalska G, Brzezinski B and Huczyński A: Synthesis, cytotoxicity and antibacterial activity of new esters of polyether antibiotic-salinomycin. Eur J Med Chem 76: 435-444, 2014.

15. Huczyński A: Polyether ionophores-promising bioactive molecules for cancer therapy. Bioorg Med Chem Lett 22: 7002-7010, 2012.

16. Todd GC, Novilla MN and Howard LC: Comparative toxicology of monensin sodium in laboratory animals. J Anim Sci 58: 1512-1517, 1984.

17. Naujokat $C$ and Steinhart R: Salinomycin as a drug for targeting human cancer stem cells. J Biomed Biotechnol 2012: 950658, 2012.

18. Scherzad A, Hackenberg S, Schramm C, Froelich K, Ginzkey C, Hagen R and Kleinsasser N: Geno- and cytotoxicity of salinomycin in human nasal mucosa and peripheral blood lymphocytes. Toxicol In Vitro 29: 813-818, 2015.

19. Scherzed A, Hackenberg S, Froelich K, Rak K, Technau A, Radeloff A, Nöth U, Koehler C, Hagen R and Kleinsasser N: Effects of salinomycin on human bone marrow-derived mesenchymal stem cells in vitro. Toxicol Lett 218: 207-214, 2013.

20. Wynn RF, Hart CA, Corradi-Perini C, O'Neill L, Evans CA, Wraith JE, Fairbairn LJ and Bellantuono I: A small proportion of mesenchymal stem cells strongly expresses functionally active CXCR4 receptor capable of promoting migration to bone marrow. Blood 104: 2643-2645, 2004.

21. Liu N, Tian J, Cheng J and Zhang J: Migration of CXCR4 gene-modified bone marrow-derived mesenchymal stem cells to the acute injured kidney. J Cell Biochem 114: 2677-2689, 2013.

22. Larzabal L, El-Nikhely N, Redrado M, Seeger W, Savai R and Calvo A: Differential effects of drugs targeting cancer stem cell (CSC) and non-CSC populations on lung primary tumors and metastasis. PloS One 8: e79798, 2013.

23. Marquez-Curtis LA and Janowska-Wieczorek A: Enhancing the migration ability of mesenchymal stromal cells by targeting the SDF-1/CXCR4 axis. BioMed Res Int 2013: 561098, 2013. 